\title{
A NUMERICAL METHOD FOR SHALLOW SHELL VIBRATION AND STABILITY PROBLEMS
}

\author{
TRAN DUC CHINH
}

Hanoi University of Civil Engineering

\section{§o. INTRODUCTION}

The stability and vibration problems of shallow shells have been studied by many scientists $[1,2]$. The usual approaches for those problems were based on the partial differential equations of high order with unknown functions being displacement $w$ and stress $\varphi$ functions. Integrating these equations by analytical method usually are too difficult because of the high order of the differential equations even if for bending problems [3].

On the base of the integral representation of displacement functions through Green's functions the author has proposed a numerical method for solving the differential equations of the problem. These equations were solved approximately after producing them into linear algebraic equations by finite difference technique.

\section{§1. GOVERNING EQUATIONS}

Vlasov's governing differential equations for thin shallow shell with variable curvatures in the form of the three displacements $(\bar{u}, \bar{v}, \bar{w})$ have been employed $[4,5]$

$$
\begin{aligned}
& L_{11}(\bar{u})+L_{12}(\bar{v})+L_{13}(\bar{w})+\frac{1-\nu^{2}}{E h}\left(X_{0}-m \frac{\partial^{2} \bar{u}}{\partial t^{2}}\right)=0 \\
& L_{21}(\bar{u})+L_{22}(\bar{v})+L_{23}(\bar{w})+\frac{1-\nu^{2}}{E h}\left(Y_{0}-m \frac{\partial^{2} \bar{v}}{\partial t^{2}}\right)=0 \\
& L_{31}(\bar{u})+L_{32}(\bar{v})+L_{33}(\bar{w})+\frac{1-\nu^{2}}{E h}\left(Z_{0}-m \frac{\partial^{2} \bar{w}}{\partial t^{2}}\right)=0
\end{aligned}
$$

where $L_{11}, L_{12}, \ldots, L_{33}$ - linear differential operators of the shell, $h$ - thickness of the shell; $X_{0}$, $Y_{0}, Z_{0}$ - harmonic surface loads situated on the shell, $m$-density of the mass for an unit area, $E$ - Young's modulus, $\nu$ - Poisson's coefficient.

For convenience in integration and computation, the dimensionless cartesian coordinates are used. In the case of free vibration $X_{0}=Y_{0}=Z_{0}=0$.

The three displacements in the governing equations are assumed in the form

$$
\begin{aligned}
\bar{u}(X, Y, t) & =u(X, Y) \sin \omega t \\
\bar{v}(X, Y, t) & =v(X, Y) \sin \omega t \\
\bar{w}(X, Y, t) & =w(X, Y) \sin \omega t
\end{aligned}
$$


Substituting the aboves into the governing equations for free vibration of the shells gives

$$
\begin{aligned}
& L_{11}(u)+L_{12}(v)+L_{13}(w)=\lambda u \\
& L_{21}(u)+L_{22}(v)+L_{23}(w)=\lambda v \\
& L_{31}(u)+L_{32}(v)+L_{33}(w)=\lambda w
\end{aligned}
$$

In the case of elastic stability the governing equations of the shell are

$$
\begin{aligned}
& L_{11}(\tilde{u})+L_{12}(\tilde{v})+L_{13}(\tilde{w})=0 \\
& L_{21}(\tilde{u})+L_{22}(\tilde{v})+L_{23}(\tilde{w})=0 \\
& L_{31}(\tilde{u})+L_{32}(\tilde{v})+L_{33}(\tilde{w})=\lambda^{*} L_{34}(\tilde{w})
\end{aligned}
$$

where operators in dimensional coordinates are $[4,5]$

$$
\begin{aligned}
& L_{11}=\frac{\partial^{2}}{\partial X^{2}}+\frac{1-\nu}{2} \frac{\partial^{2}}{\partial Y} ; \quad L_{12}=\frac{1+\nu}{2} \frac{\partial^{2}}{\partial X \partial Y} ; \quad L_{22}=\frac{\partial^{2}}{\partial Y^{2}}+\frac{1-\nu}{2} \frac{\partial^{2}}{\partial X^{2}} \\
& L_{13}=-\left(k_{1}+\nu k_{2}\right) \frac{\partial}{\partial X}-k_{12}(1-\nu) \frac{\partial}{\partial Y} ; \quad L_{23}=-\left(k_{2}+\nu k_{1}\right) \frac{\partial}{\partial Y}-k_{12}(1-\nu) \frac{\partial}{\partial X} \\
& L_{21}=L_{12} ; \quad L_{31}=L_{13} ; \quad L_{32}=L_{23} ; \\
& L_{33}=(D / C) \nabla^{4}+k_{1}^{2}+2 \nu k_{1} k_{2}+k_{2}^{2}+2(1-\nu) k_{12}^{2}
\end{aligned}
$$

with

$$
k_{1}=\frac{\partial^{2} Z}{\partial X^{2}} ; \quad k_{2}=\frac{\partial^{2} Z}{\partial Y^{2}} ; \quad k_{12}=\frac{\partial^{2} Z}{\partial X \partial Y} ;
$$

where $Z=Z(X, Y)$ - the middle surface equation of the shell;

$$
\begin{aligned}
& L_{34}=N_{x} \frac{\partial^{2}}{\partial X^{2}}+2 N_{x y} \frac{\partial^{2}}{\partial X \partial Y}+N_{y} \frac{\partial^{2}}{\partial Y^{2}} ; \quad[4,5] \\
& \lambda=-m\left(\frac{1-\nu^{2}}{E h}\right) \omega^{2} ; \quad \lambda^{*}=\frac{1-\nu}{E h} N_{c r} ; \quad D=\frac{E h^{3}}{12\left(1-\nu^{2}\right)} ; \quad C=\frac{E h}{1-\nu^{2}}
\end{aligned}
$$

\section{§2. METHOD OF ANALYSIS}

The method to be presented is based on integral representation of displacement functions through Green's functions, by which the governing differential equations of the problem are converted in to linear algebraic equations by using finite difference technique.

According to this method, the region of the shell is divided into a set of orthogonal lines $X=X_{m}(m=1, \ldots, M)$, and $Y=Y_{n}(n=1, \ldots, N)$. The highest derivaties of $u, v, w$ in eqs (1.2) and (1.3) are denoted by:

$$
\begin{array}{lll}
\frac{\partial^{2} u}{\partial X^{2}}=-k(X, Y) ; & \frac{\partial^{2} v}{\partial X^{2}}=-s(X, Y) ; & \frac{\partial^{4} w}{\partial X^{4}}=-p(X, Y) ; \\
\frac{\partial^{2} u}{\partial Y^{2}}=-d(X, Y) ; & \frac{\partial^{2} v}{\partial Y^{2}}=-t(X, Y) ; & \frac{\partial^{4} w}{\partial Y^{4}}=-q(X, Y) ;
\end{array}
$$

then, along the line $Y=Y_{n}$, eqs (2.1) can be transformed [6] to 


$$
\begin{aligned}
& u=\int_{0}^{\ell} f\left(X, \xi, Y_{n}\right) k\left(\xi, Y_{n}\right) d \xi \\
& v=\int_{0}^{\ell} e\left(X, \xi, Y_{n}\right) s\left(\xi, Y_{n}\right) d \xi \\
& w=\int_{0}^{\ell} a\left(X, \xi, Y_{n}\right) p\left(\xi, Y_{n}\right) d \xi
\end{aligned}
$$

where $f, e$ and $a$ are Green's functions associated with the homogeneous eqs of (2.1) and the boundary conditions assumed for the clamped shell as follow $u=v=w=w^{\prime}=0$ at $X=0$ and $X=\ell$.

The integral equations (2.2) can be reduced to a summation by using Simpson's rule and for the numerical integration and by using second degree interpolation $\mathcal{L}$ to relate the functions $k, s$ and $p$ at point $\left(\xi, Y_{n}\right)$ to those at points $\left(X, Y_{n}\right)$ then eqs $(2.2)$ become

$$
\begin{gathered}
u_{n}=f_{n} \alpha L_{n} k_{n}=F_{n} \cdot k_{n}, \\
v_{n}=e_{n} \alpha L_{n} s_{n}=E_{n} \cdot s_{n}, \\
w_{n}=a_{n} \alpha L_{n} p_{n}=A_{n} \cdot p_{n} .
\end{gathered}
$$

For all the lines paralleled to the $X$-axis, eqs (2.3) in matrix notation are

$$
u=F k, \quad v=E s, \quad w=A p .
$$

Similarly, eqs (2.1) can be reduced to

$$
\begin{aligned}
u & =T^{-1} H T d^{*}=\tilde{H} d ; \\
v & =T^{-1} G H t^{*}=\tilde{G} t \\
w & =T^{-1} B T q^{*}=\tilde{B} q,
\end{aligned}
$$

where * indicates the sequence of the nodal points along the lines paralleled to $X$-axis; $T$ - a unitary transformation matrix to rearrang the nodal points in the $Y$ - direction to the same order as those in the $X$ - direction.

The required derivatives of $u, v$ and $w$ in (1.2) and (1.3) are obtained by using the derivatives of Green's functions and the procedure of differential operators. For $u$, for example, the derivative are

$$
\begin{aligned}
u^{\prime} & =F^{\prime} k=F^{\prime} F^{-1} u \\
u^{\prime \prime} & =-k=-F^{-1} u ; \\
\dot{u}^{\prime} & =F^{\prime} F^{-1} \tilde{\dot{H}} \tilde{H}^{-1} u ; \\
\dot{u} & =\tilde{\dot{H}}^{-} \tilde{H}^{-1} u \\
\ddot{u} & =-d=-\tilde{H}^{-1} u .
\end{aligned}
$$

In the similar way, the derivatives for $v$ and $w$ can be obtained.

Now, we consider the shallow shell for which the middle surface equation is 


$$
Z=c\left[\frac{(X-a)^{2}}{a^{2}}+\frac{(Y-b)^{2}}{b^{2}}-\frac{(X-a)^{2}(Y-b)^{2}}{a^{2} b^{2}}-1\right] .
$$

By using the dimensionless variables $(x=X / 2 a, y=Y / 2 b)$, we obtain the differential operators of the shell as follows

$$
L_{i j}^{\prime}=4 a^{2} L_{i j}, \quad(i, j=1,2,3,4)
$$

$$
\begin{aligned}
& L_{11}^{\prime}=\frac{\partial^{2}}{\partial x^{2}}+\frac{1-\nu}{2} r^{2} \frac{\partial^{2}}{\partial y^{2}} ; \quad L_{12}^{\prime}=\frac{1+\nu}{2} r \frac{\partial^{2}}{\partial x \partial y}=L_{21}^{\prime} ; \quad L_{22}^{\prime}=r^{2} \frac{\partial^{2}}{\partial y^{2}}+\frac{1-\nu}{2} \frac{\partial^{2}}{\partial x^{2}} ; \\
& L_{23}^{\prime}=-4 r \frac{c}{a}\left\{r^{2}\left[1-(2 x-1)^{2}\right]+\nu\left[1-(2 y-1)^{2}\right]\right\} \frac{\partial}{\partial y}+8 \frac{c}{a} r(1-\nu)(2 x-1)(2 y-1) \frac{\partial}{\partial x}=L_{32}^{\prime} ; \\
& L_{13}^{\prime}=-4 \frac{c}{a}\left\{\left[1-(2 y-1)^{2}\right]+\nu r^{2}\left[1-(2 x-1)^{2}\right]\right\} \frac{\partial}{\partial x}+8 \frac{c}{a}(1-\nu)(2 x-1)(2 y-1) r^{2} \frac{\partial}{\partial y}=L_{31}^{\prime} ; \\
& L_{33}^{\prime}=-\frac{h^{2}}{48 a^{2}}\left(\frac{\partial^{4}}{\partial x^{4}}+2 r^{2} \frac{\partial^{4}}{\partial x^{2} \partial y^{2}}+r^{4} \frac{\partial^{4}}{\partial y^{4}}\right)-16\left(\frac{c}{a}\right)^{2}\left\{\left[1-(2 y-1)^{2}\right]^{2}+\right. \\
& +r^{4}\left[1-(2 x-1)^{2}\right]^{2}+2 \nu r^{2}\left[1-(2 y-1)^{2}\right]\left[1-(2 x-1)^{2}\right]+8 r^{2}(1-\nu)(2 x-1)^{2}(2 y-1)^{2} ; \\
& L_{34}^{r}=\frac{N_{x}}{N_{c r}}+2 r \frac{N_{x y}}{N_{c r}} \frac{\partial^{2}}{\partial x \partial y}+r^{2} \frac{N_{y}}{N_{c r}} \frac{\partial^{2}}{\partial y^{2}} ; \\
& \lambda=-4 a^{2} m\left(\frac{1-\nu^{2}}{E h}\right) \omega^{2} ; \quad \lambda^{*}=\frac{1-\nu^{2}}{E h} N_{c r} ; \quad r=\frac{a}{b}
\end{aligned}
$$

a. Free vibration problem

Substitution of derivatives of $u, v$ and $w$ in (1.2) and simplification will yield to eigenvalue problem

$$
[C-\lambda I]\left\{D^{*}\right\}=0
$$

where

$$
[C]=\left[\begin{array}{lll}
L_{11}^{\prime} & L_{12}^{\prime} & L_{13}^{\prime} \\
L_{21}^{\prime} & L_{22}^{\prime} & L_{23}^{\prime} \\
L_{31}^{\prime} & L_{32}^{\prime} & L_{33}^{\prime}
\end{array}\right] ; \quad\left\{D^{*}\right\}=\left\{\begin{array}{c}
u \\
v \\
w
\end{array}\right\}
$$

$$
\begin{aligned}
& L_{11}^{\prime}=-F^{-1}-\frac{1-\nu}{2} r^{2} \tilde{H}^{-1} ; \quad L_{12}^{\prime}=\frac{1+\nu}{2} r E^{\prime} E^{-1} \tilde{\dot{G}} \tilde{G}^{-1} ; \\
& L_{13}^{\prime}=-4 \frac{c}{a}\left\{\left[1-(2 y-1)^{2}\right]+\nu r^{2}\left[1-(2 x-1)^{2}\right]\right\} A^{\prime} A^{-1}+8(1-\nu) r^{2} \frac{c}{a}(2 x-1)(2 y-1) \dot{\dot{B}} \tilde{B}^{-1} ; \\
& L_{21}^{\prime}=\frac{1+\nu}{2} r F^{\prime} F^{-1} \tilde{\dot{H}} \tilde{H}^{-1} ; \quad L_{22}^{\prime}=-r^{2} \tilde{G}^{-1}-\frac{1-\nu}{2} E^{-1} ; \\
& L_{23}^{\prime}=-4 r \frac{c}{a}\left\{r^{2}\left[1-(2 x-1)^{2}\right]+\nu\left[1-(2 y-1)^{2}\right]\right\} \tilde{\dot{B}} \tilde{B}^{-1}+8 \frac{c}{a} r(1-\nu)(2 x-1)(2 y-1) A^{i} A^{-1} ; \\
& L_{31}^{\prime}=4 \frac{c}{a}\left\{\left[1-(2 y-1)^{2}\right]+\nu r^{2}\left[1-(2 x-1)^{2}\right]\right\} F^{\prime} F^{-1}-8(1-\nu) \frac{c}{a} r^{2}(2 x-1)(2 y-1) \tilde{\dot{H}} \tilde{H}^{-1} ; \\
& L_{32}^{\prime}=4 r \frac{c}{a}\left\{r^{2}\left[1-(2 x-1)^{2}\right]+\nu\left[1-(2 y-1)^{2}\right]\right\} \dot{G} \tilde{G}^{-1}-8(1-\nu) \frac{c}{a} r(2 x-1)(2 y-1) E^{\prime} E^{-1} ; \\
& L_{33}^{\prime}=-\frac{h^{2}}{48 a^{2}}\left(-A^{-1}+2 r^{2} A^{\prime \prime} A^{-1} \tilde{\dot{B}} \tilde{B}^{-1}-r^{4} \tilde{B}^{-1}\right)-16\left(\frac{c}{a}\right)^{2}\left\{\left[1-(2 y-1)^{2}\right]^{2}+\right. \\
& \left.+r^{4}\left[1-(2 x-1)^{2}\right]^{2}+2 \nu r^{2}\left[1-(2 y-1)^{2}\right]\left[1-(2 x-1)^{2}\right]+8 r^{2}(1-\nu)(2 x-1)^{2}(2 y-1)^{2}\right\} ;
\end{aligned}
$$




\section{b. The elastic stability problem}

In the similar way, (1.3) can be solved for determining the buckling loads. The differential operators $L_{i j}^{\prime}(i, j=1,2,3)$ are the same as formulated in (2.4), and:

$$
L_{34}^{\prime}=\frac{N_{x}}{N_{c r}} A^{\prime \prime} A^{-1}+2 r \frac{N_{x y}}{N_{c r}} A^{\prime} A^{-1} \tilde{\dot{B}} \tilde{B}^{-1}+r^{2} \frac{N_{y}}{N_{c r}} \tilde{\dot{B}}^{\prime} \tilde{B}^{-1} .
$$

Substituting $L_{11}^{\prime}, \ldots, L_{34}^{\prime}$ into (1.3) reduces them to linear algebraic equations:

$$
\left[C^{*}-\lambda^{*} I\right]\{\tilde{w}\}=0
$$

For non-trivial solution of $\tilde{w}$

$$
\left|C^{*}-\lambda^{*} I\right|=0
$$

where

$$
\begin{aligned}
C^{*}= & -L_{34}^{\prime}{ }^{-1} L_{31}^{\prime} L_{11}^{\prime}{ }^{-1} L_{12}^{\prime}\left(L_{22}^{\prime}-L_{21}^{\prime} L_{11}^{\prime}{ }^{-1} L_{12}^{\prime}\right)^{-1}\left(L_{21}^{\prime} L_{11}^{\prime}{ }^{-1} L_{13}^{\prime}-L_{23}^{\prime}\right)-L_{34}^{\prime}{ }^{-1} L_{11}^{\prime}{ }^{-1} L_{13}^{\prime}+ \\
& +L_{34}^{\prime}{ }^{-1} L_{32}^{\prime}\left(L_{32}^{\prime}-L_{21}^{\prime} L_{11}^{\prime}{ }^{-1} L_{12}^{\prime}\right)^{-1}\left(L_{21}^{\prime} L_{11}^{\prime}{ }^{-1} L_{12}^{\prime}\right)^{-1}\left(L_{21}^{\prime} L_{11}^{\prime}{ }^{-1} L_{13}^{\prime}-L_{23}^{\prime}\right)+L_{34}^{\prime}{ }^{-1} L_{33}^{\prime}
\end{aligned}
$$

\section{§3. RESULTS AND DISCUSSIONS}

The free vibration problem was solved for the shallow shell, the middle surface equation of which is

$$
Z=c\left[\frac{(X-a)^{2}}{a^{2}}+\frac{(Y-b)^{2}}{b^{2}}+\frac{(X-a)^{2}(Y-b)^{2}}{a^{2} b^{2}}-1\right]
$$

The present results are based on the following dimensions and properties of the shell $a=b=$ $22.8 \mathrm{~cm}, h=0.1587 \mathrm{~cm}, E=3.3 \cdot 10^{2} \mathrm{KN} / \mathrm{cm}^{2}, \nu=0.4$. The form of Green's functions $f, e$ and $a$ was given by Korenev B. G. [6].

The convergence of the solution for free vibrations was shown in Table 1 . It is obvious that the

\begin{tabular}{|c|c|c|c|c|}
\hline \multirow{3}{*}{$\begin{array}{c}\text { Mesh } \\
\frac{N \times N}{\text { Mode }}\end{array}$} & \multicolumn{4}{|c|}{$r=a / b=1.0$} \\
\hline & \multicolumn{2}{|c|}{$c / h=5$} & \multicolumn{2}{|c|}{$c / h=16$} \\
\hline & 1 st mode & $2 \mathrm{nd}$ mode & 1 st mode & 2 nd mode \\
\hline $3 \times 3$ & 28.031 & 28.031 & 70.476 & 70.476 \\
\hline $5 \times 5$ & 57.333 & 40.419 & 69.677 & 72.204 \\
\hline $7 \times 7$ & 41.288 & 41.822 & 72.608 & 73.904 \\
\hline $9 \times 9$ & 40.865 & 42.171 & 49.543 & .81 .466 \\
\hline $11 \times 11$ & 40.793 & 41.924 & 82.988 & 83.427 \\
\hline $13 \times 13$ & 40.815 & 42.210 & 83.526 & 84.122 \\
\hline
\end{tabular}
convergence is more rapid for low ratio $(c / h=5)$ than for higher ratio $(c / h=16)$. It is found that the main factor affecting on the convergence are the mesh size, the rise of thickness ratio, boundary condictions and the degree of Green's function used in the solution. In Table 2 the comparison of the results of minimum natural frequency of the shell with Galerkin's solution was given.

Table 1

Remarks : 1 st mode - symmetrical in $x$ and $y$ directions; 2 nd mode - antisymmetrical in $x$ and $y$ directions; Multiplier $\left(1 / a^{2}\right) \sqrt{D / M}$. 
Table 2

\begin{tabular}{|c|c|c|}
\hline Case & Method & $\omega$ \\
\hline$c / h=0$ & Present meth. & 9.0042 \\
\hline$a / b=1$ & Galerkine's meth. [2] & 9.0359 \\
\hline$c / h=5$ & Present meth. & 22.536 \\
\hline$a / b=0.5$ & Galerkine's meth. $[2]$ & 26.985 \\
\hline$c / h=5$ & Present meth. & 40.815 \\
\hline$a / b=1.0$ & Galerkine's meth. [2] & 42.501 \\
\hline$c / h=10$ & Present meth. & 61.053 \\
\hline$a / b=1.0$ & Galerkine's meth. $[2]$ & 81.294 \\
\hline$c / h=16$ & Present meth. & 83.426 \\
\hline$a / b=1$ & Galerkine's meth. [2] & 133.255 \\
\hline \multicolumn{2}{|c|}{ Multiplier $\left(1 / a^{2}\right) \sqrt{D / M}$} & \\
\hline
\end{tabular}

Acknowledgement. The author would like to thank Prof. Dr. Sc. Dao Huy Bich for useful remarks and discussion about obtained results.

\section{REFERENCES}

1. Volmir A. S. The stability of deformable system. Nauka, M. 1975 (in Russian).

2. Onyashvili V. D. The Vlasov's theory applied to shallow shell vibration problems. Gosstroiizdat, M. 1950 (in Russian).

3. Tran Duc Chinh. The generalized systems method applied to shallow anisotropic shell bending problem. Proceedings of the 5 th National Conference on Mechanics, Hanoi, 1993 (in Vietnamese).

4. Mileykovsky I. E. The practical methods applied to shallow shell bending problems. Stroiizdat, M. 1979 (in Russian).

5. Vlasov V. Z. The general theory of shells and its applications to technology. Gostekhizdat, M. 1949 (in Russian).

6. Korenev B. G. Some applications of the Green's function theory in mathematical physics and mechanics of constructions. Fizmatizdat, M. 1965 (in Russian).

Received April 21, 1999

\section{MỘT PHƯƠNG PHÁP SỐ GIẢI BÀI TOÁN DAO ĐộNG VA ỔN ĐỊNH CƯA VỎ THOẢI}

Trên cơ sở biểu diễn tích phân các hàm chuyển vị thông qua các hàm Green, tác giả đã kiến nghị một phương pháp số để giải hệ phương trình vi phân của bài toán. Các phương trình này đã được giải gần đúng sau khi đưa chúng về hệ phương trình đại số tưyến tính nhờ kỹ thuật sai phân hữu hạn theo lược đồ Xamarsky A. A. Đã giải một số ví dụ băng số cho bài toán tìm tần số dao động riêng của vó thoải với phương trình mặt dạng paraboloit và so sánh với nghiệm thu bới Onyashvili $\mathrm{O}$. D. bằng phương pháp Galerkin [2]. 